\title{
STRICTLY REAL BANACH ALGEBRAS
}

\author{
JOHN BORIS MILLER
}

\begin{abstract}
A complex Banach algebra is a complexification of a real Banach algebra if and only if it carries a conjugation operator. We prove a uniqueness theorem concerning strictly real selfconjugate subalgebras of a given complex algebra. An example is given of a complex Banach algebra carrying two distinct but commuting conjugations, whose selfconjugate subalgebras are both strictly real. The class of strictly real Banach algebras is shown to be a variety, and the manner of their generation by suitable elements is proved. A corollary describes some strictly real subalgebras in Hermitian Banach star algebras, including $C^{*}$ algebras.
\end{abstract}

\section{INTRODUCTION}

A real Banach algebra $\mathfrak{A}$ is called strictly real if each of its elements has a real spectrum. The terminology goes back at least to Ingelstam [2]. Relying upon a 1949 theorem of Kaplansky's, it was shown in [5] and [6] that such an algebra carries a partial order $\leqslant$ and a preorder $\preceq$, compatible with the algebra structure and having many convenient and useful properties. The definitions of the orders are:

$$
x<y \text { means } \operatorname{Sp}(y-x) \subset \mathbb{R}^{+} \backslash\{0\} ; x \preceq y \text { means } \operatorname{Sp}(y-x) \subset \mathbb{R}^{+}
$$

Their presence enables quite a lot of analysis of real type to be done in $\mathfrak{A}$. For instance, the theory of the exp and Log and root functions is simpler than for more general Banach algebras, and the classical inequalities (of the mean, Hölder's, Minkowski's,...) all hold in appropriate circumstances. The resolvent is a Pick function on its domain. There are integral representation theorems in the dual space. See $[5,6]$.

In view of this theory, it is apposite to study strictly real Banach algebras as a class, in the hope of locating them more clearly within the wider class of Banach algebras, real and complex. The early theorems in this connection are due to Ingelstam. In this paper we give a number of such results. After some preliminaries in this section, Section 2 deals with complexification and conjugation, culminating after some propositions of independent interest in Theorem 2.10, a partial uniqueness result in answer to the question: Can a complex Banach algebra be simultaneously the complexification of

Received 17 June 1992

Copyright Clearance Centre, Inc. Serial-fee code: 0004-9729/93 \$A2.00+0.00. 
more than one strictly real Banach algebra? In Section 3 some examples are given, including the one mentioned in the abstract.

Section 4 has two main results: Theorem 4.4 which shows that strictly real Banach algebras form a variety; and Theorem 4.7 concerning generation of strictly real subalgebras. Connection is made with $C^{*}$ algebras.

We summarise the notation and some background facts. For any two nonempty sets $A$ and $B$ of numbers, or algebra elements, $A+B:=\{a+b: a \in A, b \in B\}$ and $A B:=\{a b: a \in A, b \in B\}$. Throughout this paper, Banach algebra means Banach algebra with identity, usually written $e$. For a real Banach algebra $\mathfrak{A}$ and $x \in \mathfrak{A}$, the spectrum of $\boldsymbol{x}$ in $\mathfrak{A}$, written $\mathrm{S}_{\mathfrak{A}}(\boldsymbol{x})$, is by definition the spectrum of $\boldsymbol{x}$ as an element of $\mathfrak{A}_{\mathbb{C}}$, the complexification of $\mathfrak{A}$, written $\mathrm{S}_{\mathfrak{A}_{\mathbb{C}}}(x)$; see Section 2. Suffixes may be omitted when the algebra is obvious. The spectral radius function is written $\nu ; \operatorname{Res}(x)$ denotes $\mathbb{C} \backslash \operatorname{Sp}(x)$. Let $\mathfrak{R}$ be the radical of $\mathfrak{A}$, that is, the intersection of all maximal left ideals of $\mathfrak{A}$; we write $\mathfrak{A}^{\prime}$ for $\mathfrak{A} \bmod \mathfrak{R}$, with elements $\boldsymbol{x}^{\prime}=\boldsymbol{x}+\mathfrak{R}$; and similarly for $\mathfrak{A}_{\mathbb{C}}$ with radical $\mathfrak{R}_{\mathfrak{C}}$. We have $\mathrm{Sp}_{\mathfrak{A}^{\prime}}\left(\boldsymbol{x}^{\prime}\right)=\mathrm{S}_{\mathrm{P}_{\mathfrak{A}}}(x)$. Kaplansky's theorem [4] tells us that if $\mathfrak{A}$ is strictly real then $\mathfrak{A}^{\prime}$ is commutative. From this it follows for strictly real $\mathfrak{A}$ that

$$
\mathrm{Sp}(x+y) \subseteq \mathrm{Sp}(x)+\mathrm{Sp}(y), \quad \mathrm{Sp}(x y) \subseteq \mathrm{Sp}(x) . \mathrm{Sp}(y)
$$

for all $x, y \in \mathfrak{A}$; and moreover $\mathfrak{R}$ coincides with the set of quasinilpotents of $\mathfrak{A}$. Inclusions (1.2) ensure the convenient properties of the orders (1.1). For the details see [5]. It is proved in Section 2 below that analogous results hold for $\mathfrak{A}_{\mathbb{C}}$; this is an important technical step.

\section{COMPLEXIFICATION AND CONJUGATION}

We recall the notion of algebra complexification of a real algebra $\mathfrak{A}$ : it is the algebra $\mathfrak{B}:=\mathfrak{A} \times \mathfrak{A}$, in which the operations are defined in the obvious way $((a, b)+(c, d):=(a+c, b+d) ;(\alpha+i \beta)(a, b):=(\alpha a-\beta b, \beta a+\alpha b) ;(a, b)(c, d)$ $:=(a c-b d, b c+a d))$, so that the identification $a \mapsto(a, 0)$ embeds $\mathfrak{A}$ monomorphically in $\mathfrak{B}$ and allows us to write $(a, b)=a+i b$ (where $i:=(0,1)$ ). $\mathfrak{A}$ is then identified with its copy in $\mathfrak{B}$, as a real subalgebra, and $\mathfrak{B}=\mathfrak{A}+\mathfrak{i A}$. (This sum is direct only in the category of real vector spaces.)

Suppose that $\mathfrak{A}$ is a real Banach algebra, with norm $\|\cdot\|$. Well-known and nontrivial theory shows that $\mathfrak{B}$ can be given a norm, 1 . I say, such that

(i) 1.1 makes $\mathfrak{B}$ a complex Banach algebra, and

(ii) 1.1 relativises to $\|$.$\| on \mathfrak{A}$.

See Rickart [7, p.5-9] (where on p.8, $\|(a, b)\|$ denotes what we call $|a+i b|$ ), Bonsall and Duncan [1, p.68-70]. It is shown in [7] that the norm is unique to within equiva- 
lence, that is, if $1.1^{\prime}$ is any other algebra norm on $\mathfrak{B}$ with properties (i) and (ii) then 1.1 and $1 . l^{\prime}$ are equivalent. Moreover, for all $a, b \in \mathfrak{A}$,

$$
|a+i b| \leqq\|a\|+\|b\| \leqq k|a+i b|
$$

(where $k=2 \sqrt{2}$ in [7], 2 in [1]), and it can be verified that

$$
|a+i b|=|a-i b| .
$$

We shall use the term Banach complexification of $\mathfrak{A}$ for $\mathfrak{B}$ normed by any norm 1 . $\boldsymbol{I}^{\prime}$ with properties (i), (ii), (2.1) for some positive constant $k$, and (2.2); the standard Banach complexification will mean $\mathfrak{B}$ normed by $\mathbf{I} .1$. (Even $\mathbf{I} I$ is not uniquely determined in the discussion in [7], since it depends upon the choice of an arbitrary real Banach space, there called $\mathfrak{X}$. To make the definition of $|$.$| specific, we shall require$ that $\mathfrak{X}$ is $\mathfrak{A}$.) The standard Banach complexification of $\mathfrak{A}$ will be denoted by $\mathfrak{A}_{\mathfrak{C}}$.

For any complex algebra $\mathfrak{B}$, let $\mathbb{R} \mathfrak{B}$ denote the real algebra which is $\mathfrak{B}$ with $\mathbb{R}$ as its scalar field. The term 'real subalgebra of $\mathfrak{B}^{\prime}$ shall mean, as usual, subalgebra of $\mathbb{R} \mathfrak{B}$.

Again, let us start with a real Banach algebra $\mathfrak{A}$, and its standard Banach complexification $\mathfrak{A}_{\mathbb{C}}$. Since each element of $\mathfrak{A}_{\mathbb{C}}$ is uniquely representable in the form $a+i b$, $a, b \in \mathfrak{A}$, there exists the canonical conjugation operator $c$ on $\mathfrak{A}_{\mathfrak{C}}$, defined by

$$
(a+i b) \mathrm{c}=a-i b .
$$

The operator satisfies, for all $x, y \in \mathfrak{A}_{\mathbb{C}}$ and $\lambda \in \mathbb{C}$

(1) $(x+y) c=x c+y c$

(2) $(\lambda x) c=\bar{\lambda}(x c)$,

(3) $(x y) c=x c y c$

(4) $x \mathrm{cc}=x$,

(5) $\|x \mathrm{c}\|=\|x\|$,

and

$$
x \mathrm{c}=\boldsymbol{x} \text { if and only if } x \in \mathfrak{A} .
$$

Here $\bar{\lambda}$ in (2) denotes the complex conjugate of $\lambda ;(5)$ is the property (2.2). We draw attention to (3), where there is no reversal of order of the factors.

More generally, let $\mathfrak{B}$ be any complex Banach algebra. Definition: any operator $c: \mathfrak{B} \rightarrow \mathfrak{B}$ having properties (1) to (5) will be called a conjugation on $\mathfrak{B}$. By (4) it is a surjection. If $\mathfrak{B}$ is commutative, but not otherwise, the notion coincides with that of isometric involution, about which there is a large literature. Given $c$ on $\mathfrak{B}$, write

$$
\mathfrak{A}:=\{x \in \mathfrak{B}: x \mathbf{c}=x\} \text {. }
$$


This defines a real Banach subalgebra of $\mathfrak{B}$ : that $\mathfrak{A}$ is closed in $\mathfrak{B}$ is a consequence of (5). Moreover, since $c$ is onto, the identity $e$ belongs to $\mathfrak{A}$. Note that (3) makes $\mathfrak{A}$ a ring, not merely a real vector subspace as happens with involutions. $\mathfrak{A}$ will be called the selfconjugate subalgebra with respect to $c$.

If $c$ satisfies (1) to (4) and is continuous, $\mathfrak{B}$ can be renormed by the equivalent norm $\|x\|=\max \{\|x\|,\|x c\|\}$, which satisfies (5) and relativises to $\|$.$\| on \mathfrak{A}$. In fact, in the ensuing theory it often suffices to weaken the definition of conjugation in this way, replacing (5) by

(5') $c$ is continuous.

THEOREM 2.1. Let $\mathfrak{B}$ be any complex Banach algebra, $c$ a conjugation on $\mathfrak{B}$, and $\mathfrak{A}$ its selfconjugate subalgebra. Then $\mathfrak{B}$ is a Banach complexification of $\mathfrak{A}$, and $c$ is the canonical conjugation operator on $\mathfrak{B}$ with respect to $\mathfrak{A}$, so that $\mathfrak{B}$ is equivalent to $\mathfrak{A}_{\mathbb{C}} \cdot \mathfrak{A}$ is maximal among all real subalgebras $\mathfrak{E}$ of $\mathfrak{B}$ for which $\mathfrak{E} \cap \mathfrak{E}=\{0\}$.

Proof: The norm on $\mathfrak{A}$ is the norm of $\mathfrak{B}$ relativised. For $x \in \mathfrak{B}$ write $a=$ $(x+x c) / 2$ and $b=i(x \mathrm{c}-x) / 2$. Then $a, b \in \mathfrak{A}$, and

$$
x=a+i b, \quad x \mathrm{c}=a-i b .
$$

This representation of $x$ is unique, for if $x=p+i q$ with $p, q \in \mathfrak{A}$ then necessarily $p=a$ and $q=b$. Thus $\mathfrak{B}$ is the algebraic complexification of $\mathfrak{A}$. Properties (i) and (ii) clearly hold on $\mathfrak{B}$, and (2.2) holds since $c$ satisfies (5) and (2.5). Finally, (2.1) holds, for with $a, b \in \mathfrak{A}$ we have

$$
\|a\|+\|b\|=\left\|\frac{1}{2}(x+x c)\right\|+\left\|\frac{1}{2}(x-x c)\right\| \leqslant\|x\|+\|x c\|=2\|a+i b\| .
$$

Thus $(\mathfrak{B},\|\cdot\|)$ is a Banach complexification of $(\mathfrak{A},\|\cdot\|)$; and (2.5) shows that $c$ is the canonical conjugation. The maximality property is easily verified.

Corollary 2.2. A necessary and sufficient condition for a complex Banach algebra to be a Banach complexification of some real Banach algebra is that there exist on it a conjugation.

The theorem also shows that any theorem about an arbitrary real Banach algebra $\mathfrak{A}$ and its complexification $\mathfrak{A}_{\mathbb{C}}$ can, to within equivalence of norms, be reformulated as a theorem about a complex Banach algebra $\mathfrak{B}$ carrying a conjugation $c$, and its selfconjugate subalgebra $\mathfrak{A}$; and vice versa. In later theorems we shall therefore opt for whichever formulation seems the more convenient.

We remark that elements of the forms $(x \mathrm{c}) x$ and $x(x \mathrm{c})$ need not belong to $\mathfrak{A}$.

As one might suppose, there is a simple connection between conjugations and isometric involutions, by means of the notion of transposition. We define a transposition 
on $\mathfrak{B}$ to be a linear operator $t$ on $\mathfrak{B}$ which is involutory ( $t \mathfrak{t}=$ identity), isometric $(\|x t\|=\|x\|)$ and reverses products $((x y) t=y t x t)$. Let $C, I$ and $T$ denote the sets of all conjugations, isometric involutions and transpositions on $\mathfrak{B}$ respectively. If $f$ and $g$ are any two elements from two distinct such sets, and $f$ and $g$ commute, then $f g$ belongs to the third set.

We turn to the question of distinct conjugations on the same algebra $\mathfrak{B}$. For a given conjugation $f$ we denote its selfconjugate subalgebra of $\mathfrak{B}$ by $\mathfrak{A}_{\mathfrak{f}}$.

Lemma 2.3. The function $f \mapsto \mathfrak{A}_{\mathfrak{f}}$ is one-one. More precisely, if $\mathrm{f} \neq \mathrm{g}$ then neither of $\mathfrak{A}_{\mathrm{f}}$ and $\mathfrak{A}_{\mathrm{g}}$ is contained in the other.

Proof: Suppose $\mathfrak{A}_{\mathfrak{f}}=\mathfrak{A}_{\mathrm{g}}$. Let $x \in \mathfrak{B}$, and let $x=a+i b$ be its unique representation with respect to $f$. Then $x \mathrm{f}=a-i b$; but $a, b \in \mathfrak{A}_{g}$, so $x=a+i b$ is also the unique representation with respect to $g$ and therefore $x \mathrm{~g}=a-i b$. Thus $f=g$.

The second assertion of the theorem follows from the maximality assertion in Theorem 2.1, or from the following elementary argument. Suppose that $\mathfrak{A}_{\mathfrak{1}} \varsubsetneqq \mathfrak{A}_{8}$. There exists $y$ such that $y \mathrm{f} \neq y=y \mathrm{~g}$. The element $z=(y+y \mathrm{f}) / 2$ satisfies $z \mathrm{f}=z$, so $z \in \mathfrak{A}_{\mathrm{r}}$. Hence $z \mathrm{~g}=z$, which gives

$$
y \mathrm{~g}+y \mathrm{fg}=y+y \mathrm{f} .
$$

Likewise $w=i(y \mathrm{f}-y) / 2 \in \mathfrak{A}_{\mathrm{g}}$ so

$$
-y \mathrm{~g}+y \mathrm{f} g=y-y \mathrm{f} .
$$

From these equations we deduce that $y \mathrm{f}=\boldsymbol{y g}$, contradiction.

Lemma 2.4. For all $x$ in $\mathfrak{B}, \operatorname{Sp}(x \mathrm{c})=\{\bar{\lambda}: \lambda \in \operatorname{Sp}(x)\}$, and for $\lambda \in \operatorname{Res}(x \mathrm{c})$,

$$
(\lambda e-x c)^{-1}=\left((\lambda e-x)^{-1}\right) \mathrm{c} \text {. }
$$

So $\operatorname{Sp}(x \mathrm{c})$ is independent of the choice of conjugation c. For $z \in \mathfrak{A}, \operatorname{Sp}(a)$ is symmetrical about the real axis.

The result is well known, and its proof is straightforward.

Lemma 2.5. Let $\mathfrak{A}$ be strictly real. Then for $a, b \in \mathfrak{A}$, the spectrum of $a+i b$ in $\mathfrak{B}$ is contained in the product in $\mathbb{R}^{2}$ of the spectra of $a$ and $b$ :

$$
\mathrm{S}_{\mathrm{P}_{\mathfrak{B}}}(a+i b) \subseteq \mathrm{S}_{\mathfrak{A}}(a)+i \mathrm{SP}_{\mathfrak{A}}(b) .
$$

Proof: First we show that if either of $a$ or $b$ is invertible in $\mathfrak{A}$ then $a+i b$ is invertible in $\mathfrak{B}$. Suppose $a$ is invertible and write $x=a+b a^{-1} b$. By commutativity of $\mathfrak{A}^{\prime}$,

$$
\begin{aligned}
\operatorname{Sp}_{\mathfrak{A}}(x) & =\operatorname{Sp}_{\mathfrak{A}^{\prime}}\left(x^{\prime}\right) \subseteq \mathrm{S}_{\mathrm{P}_{\mathfrak{A}^{\prime}}}\left(a^{\prime 2}+b^{\prime 2}\right) \cdot \mathrm{S}_{\mathrm{P}_{\mathfrak{A}}}\left(a^{\prime}-1\right) \\
& =\mathrm{S}_{\mathrm{P}_{\mathfrak{A}}}\left(a^{2}+b^{2}\right) \cdot \mathrm{Sp}_{\mathfrak{A}}\left(a^{-1}\right) .
\end{aligned}
$$


Now $\operatorname{Sp}\left(a^{2}\right) \subset \mathbb{R}^{+} \backslash\{0\}, \operatorname{Sp}\left(b^{2}\right) \subset \mathbb{R}^{+}$so $\operatorname{Sp}\left(a^{2}+b^{2}\right) \subset \mathbb{R}^{+} \backslash\{0\} ;$ therefore $0 \notin$ $\mathrm{Sp}_{\mathfrak{a}}(\boldsymbol{x}), \boldsymbol{x}$ is invertible. Write

$$
u=x^{-1}, \quad v=-a^{-1} b x^{-1}
$$

Then $a u-b v=e, b u+a v=0$, so $(a+i b)(u+i v)=e$. Thus $a+i b$ has a right inverse; similarly it has a left inverse; therefore it is invertible. The same outcome follows if instead we assume $b$ invertible.

Now suppose instead that $\lambda \in \operatorname{Res}(a) \cap \mathbb{R}$; it follows that for any real $\beta,(\lambda e-a)+$ $i(\beta e-b)$ is invertible in $\mathfrak{B}$, and similarly if $\mu \in \operatorname{Res}(b) \cap \mathbb{R}$ then $(\beta e-a)+i(\mu e-b)$ is invertible. Hence if $\lambda+i \mu \in \mathrm{Sp}_{\mathfrak{x}}(a+i b)$, then $\lambda \in \mathrm{Sp}_{\mathfrak{a}}(a)$ and $\mu \in \mathrm{Sp}_{\mathfrak{A}}(b)$.

We wish to show that $S_{p_{\mathfrak{B}}}$ has the same properties (1.2) as $S_{p_{\mathfrak{A}}}$. To this end it is necessary to find the connection between the radicals $\mathfrak{R}_{\mathfrak{A}}$ and $\mathfrak{R}_{\mathfrak{B}}$ of $\mathfrak{A}$ and $\mathfrak{B}$ respectively, in order to prove that $\mathfrak{B} \bmod \mathfrak{R}_{\mathfrak{B}}$, like $\mathfrak{A}^{\prime}$, is commutative when $\mathfrak{A}$ is strictly real.

THEOREM 2.6. If $\mathfrak{A}$ is strictly real, then $\mathfrak{R}_{\mathfrak{B}}=\mathfrak{R}_{\mathfrak{A}}+i \mathfrak{R}_{\mathfrak{A}}$.

Proof: A subset $L$ of $\mathfrak{B}$ is called selfconjugate if $L \mathrm{c}=L$. If $\mathrm{L}$ is any left ideal of $\mathfrak{B}$, write

$$
L_{0}=\{a \in \mathfrak{A}: a+i b \in L \text { for some } b \in \mathfrak{A}\} .
$$

It is clear that $L \subseteq L_{0}+i L_{0}$, and $L_{0}+i L_{0}$ is a selfconjugate left ideal.

Suppose that $L$ is a maximal left ideal of $\mathfrak{B}$; then either $L_{0}+i L_{0}=\mathfrak{B}$, or $L_{0}+i L_{0}=L$. If the former, then $e \in L_{0}+i L_{0}$, that is, $e \in L_{0}$, so there exists $b \in \mathfrak{A}$ such that $e+i b \in L$. But $e+i b$ is invertible by Lemma 2.5; this contradiction shows that $L_{0}+i L_{0}=L$. Moreover it is not difficult to deduce that $L_{0}$ is a maximal left ideal of $\mathfrak{A}$.

Conversely, if $M$ is any maximal left ideal of $\mathfrak{A}$ then $K:=M+i M$ is a maximal selfconjugate left ideal of $\mathfrak{B}$. Let $K \subseteq N$ for some maximal left ideal $N$ of $\mathfrak{B}$. Then $N=N_{0}+i N_{0}$, so $M \subseteq N_{0}$, whence $M=N_{0}$, and $N=M+i M=K$.

Thus we see that every maximal left ideal $N$ of $\mathfrak{B}$ is selfconjugate and of the form $N=M+i M$ where $M$ is a maximal left ideal of $\mathfrak{A}$; and conversely if $M$ is any maximal left ideal of $\mathfrak{A}$ then $N$ so defined is a maximal left ideal of $\mathfrak{B}$. Therefore

$$
\begin{aligned}
\mathfrak{R}_{\mathfrak{B}} & =\bigcap\{M+i M: M \text { is a maximal left ideal of } \mathfrak{A}\} \\
& =\bigcap_{M} M+i \bigcap_{M} M=\mathfrak{R}_{\mathfrak{A}}+i \mathfrak{R}_{\mathfrak{A}}
\end{aligned}
$$


COROLlary 2.7. When $\mathfrak{A}$ is strictly real,

$$
\mathfrak{B} \bmod \mathfrak{R}_{\mathfrak{B}} \cong \mathfrak{A} \bmod \mathfrak{R}_{\mathfrak{A}}+\mathfrak{i} \mathfrak{A} \bmod \mathfrak{R}_{\mathfrak{A}},
$$

$\mathfrak{B} \bmod \mathfrak{R}_{\mathfrak{B}}$ is commutative, and $\mathfrak{R}_{\mathfrak{B}}$ coincides with the set of all quasinilpotents of $\mathfrak{B}$. Also $\mathfrak{R}_{\mathfrak{A}}=\mathfrak{A} \cap \mathfrak{R}_{\mathfrak{B}}$.

The typical member of the lefthand side of (2.7) is of the form

$$
(a+i b)+\mathfrak{R}_{\mathfrak{A}}+i \mathfrak{R}_{\mathfrak{A}}=\left(a+\mathfrak{R}_{\mathfrak{A}}\right)+i\left(b+\mathfrak{R}_{\mathfrak{A}}\right),
$$

so the isomorphism is actually logical identity of the algebras; we do not assert the equality of the norms. Equation (2.7) can be written more briefly as

$$
\left(\mathfrak{A}_{\mathbb{C}}\right)^{\prime} \cong\left(\mathfrak{A}^{\prime}\right)_{\mathbb{C}}
$$

COROLlaRY 2.8. If $\mathfrak{A}$ is strictly real, $\mathrm{Sp}_{\mathfrak{B}}$ has the properties (1.2) on $\mathfrak{B}$.

COROLlaRY 2.9. If $\mathfrak{A}$ is strictly real, $a+i b$ is invertible in $\mathfrak{B}$ if and only if $a^{2}+b^{2}$ is invertible in $\mathfrak{A}$.

PROOF: If $a^{2}+b^{2}$ is invertible, we have

$$
\begin{aligned}
(a+i b)(a-i b) & =a^{2}+b^{2}+i(b a-a b) \\
& =\left(a^{2}+b^{2}\right)\left[e+i\left(a^{2}+b^{2}\right)^{-1}(b a-a b)\right]
\end{aligned}
$$

here $i\left(a^{2}+b^{2}\right)^{-1}(b a-a b)$ belongs to $i \Re_{\mathfrak{A}}$ and hence to $\Re_{\mathfrak{B}}$, and is therefore quasinilpotent, by Corollary 2.7. Hence the second factor on the right is invertible, showing that $a+i b$ has a right inverse. Similarly it has a left inverse, and so is invertible. Conversely, if $a+i b$ is invertible then so is $a-i b$, by Lemma 2.4, and the invertibility of $a^{2}+b^{2}$ follows.

The main result of this section is an assertion concerning the partial uniqueness of strictly real subconjugate subalgebras: namely that, modulo the radical, and within any one class of pairwise commuting conjugations, there is at most one strictly real selfconjugate subalgebra of a given complex Banach algebra.

ThEOREM 2.10. Let $\mathfrak{B}$ be a complex Banach algebra, with radical $\mathfrak{R}$, and let $f$ and $g$ be commuting conjugations on $\mathfrak{B}$ for which $\mathfrak{A}_{\mathrm{f}}$ and $\mathfrak{A}_{\mathrm{g}}$ are strictly real. If $\mathfrak{R}_{\mathrm{f}}$ and $\mathfrak{R}_{\mathrm{g}}$ denote the radicals of $\mathfrak{A}_{\mathfrak{f}}$ and $\mathfrak{A}_{\mathrm{g}}$ respectively, then

$$
\mathfrak{A}_{\mathrm{f}}=\mathfrak{A}_{\mathrm{f}} \cap \mathfrak{A}_{\mathrm{g}}+\mathfrak{R}_{\mathrm{f}}, \quad \mathfrak{A}_{\mathrm{g}}=\mathfrak{A}_{\mathrm{f}} \cap \mathfrak{A}_{\mathrm{g}}+\mathfrak{R}_{\mathrm{g}},
$$

and

$$
\mathfrak{A}_{\mathrm{f}}+i \mathfrak{R}_{\mathrm{f}}=\mathfrak{A}_{\mathrm{g}}+i \mathfrak{R}_{\mathrm{g}}=\{x \in \mathfrak{B}: \mathrm{Sp}(\boldsymbol{x}) \text { is real }\}
$$


Also the algebras $\mathfrak{A}_{\mathfrak{f}} \bmod \mathfrak{R}_{\mathrm{f}}$ and $\mathfrak{A}_{\mathrm{g}} \bmod \mathfrak{R}_{\mathrm{g}}$ are isomorphic.

If $\mathrm{f} \neq \mathrm{g}$ then neither of $\mathfrak{R}_{\mathrm{f}}, \mathfrak{R}_{\mathrm{g}}$ contains the other. We have $\mathfrak{R}_{\mathrm{f}} \cap \mathfrak{R}_{\mathrm{g}}=\{0\}$ if and only if $\mathfrak{R}_{\mathrm{f}}=i \mathfrak{R}_{\mathrm{g}}$.

If $\mathfrak{A}_{\mathfrak{f}}$ or $\mathfrak{A}_{\mathrm{g}}$ is semisimple, in particular if $\mathfrak{B}$ is semisimple, then $f=g$.

Proof: First, we remark that if $y \in \mathfrak{A}_{\mathrm{g}}$ then also $y \mathrm{f} \in \mathfrak{A}_{\mathfrak{g}}$, because of commutativity. Next, observe that if $x \in \mathfrak{A}_{\mathfrak{f}}$ and

$$
x=a+i b \text { with } a, b \in \mathfrak{A}_{\mathrm{g}},
$$

is its canonical representation with respect to $\mathrm{g}$, then $a, i b \in \mathfrak{A}_{\mathfrak{f}}$. For

$$
x=x \mathrm{f}=a \mathrm{f}-i b \mathrm{f} \text {, so } x \mathrm{~g}=a \mathrm{~g} \mathrm{f}+i b \mathrm{gf}=a \mathrm{f}+i b \mathrm{f} \text {. }
$$

But here $a \mathrm{f}, b \mathrm{f} \in \mathfrak{A}_{\mathrm{g}}$, by the initial remark. Thus $x \mathrm{~g}=a \mathrm{f}+i b \mathrm{f}$ is the canonical representation of $x \mathrm{~g}$ with respect to $\mathrm{g}$, which is also $x \mathrm{~g}=a-i b$. Therefore $a=a \mathrm{f}$, $b=-b \mathrm{f}$, whence $a, i b \in \mathfrak{A}_{\mathrm{f}}$, as asserted. Hence $x-a \in \mathfrak{A}_{\mathfrak{f}}$, and since $\mathfrak{A}_{\mathfrak{f}}$ is strictly real and $\mathfrak{B}$ is a Banach complexification of $\mathfrak{A}_{\mathfrak{f}}$, we have

$$
\mathrm{Sp}_{\mathfrak{B}}(x-a)=\mathrm{Sp}_{\mathfrak{x}_{\mathfrak{f}}}(x-a) \subset \mathbb{R} \text {. }
$$

Similarly

$$
\mathrm{SP}_{\mathfrak{B}}(i b)=i \mathrm{~S}_{\mathrm{P}_{\mathfrak{B}}}(b)=i \mathrm{SP}_{\mathbf{P}_{\mathfrak{g}}}(b) \subset i \mathbb{R} ;
$$

therefore $\mathrm{Sp}_{\mathfrak{B}}(x-a)=\{0\}=\mathrm{Sp}_{\mathfrak{B}}(i b), x-a, i b \in \mathfrak{R}$, in fact $i b \in \mathfrak{A}_{\mathfrak{R}} \cap \mathfrak{R}=\mathfrak{R}_{\mathfrak{f}}$. Since $x$ was arbitrary in $\mathfrak{A}_{\mathfrak{f}},(2.11)$ shows that $\mathfrak{A}_{\mathfrak{f}} \subseteq \mathfrak{A}_{\mathfrak{f}} \cap \mathfrak{A}_{\mathrm{g}}+\mathfrak{R}_{\mathfrak{f}}$. Since the righthand side here is contained in $\mathfrak{A}_{f}$, there is equality; this is the first equation of (2.9), the second follows by symmetry.

Equation (2.11) also gives $\mathfrak{A}_{\mathrm{f}} \subseteq \mathfrak{A}_{\mathrm{g}}+\mathfrak{R}$, and since the righthand side here also contains $\mathfrak{R}$,

$$
\mathfrak{A}_{\mathfrak{f}}+\mathfrak{R}=\mathfrak{A}_{\mathrm{g}}+\mathfrak{R} .
$$

Putting on the left $\mathfrak{R}=\mathfrak{R}_{\mathfrak{f}}+\mathfrak{i} \mathfrak{R}_{\mathfrak{f}}$ from Theorem 2.6 and using $\mathfrak{A}_{\mathfrak{f}}+\mathfrak{R}_{\mathrm{f}}=\mathfrak{A}_{\mathfrak{f}}$, and similarly on the right, we get the first part of (2.10). The second comes by an application of Corollary 2.8 .

To prove $\mathfrak{A}_{\mathrm{f}}^{\prime} \cong \mathfrak{A}_{\mathrm{g}}^{\prime}$, let $\boldsymbol{x}^{\prime} \in \mathfrak{A}_{\mathrm{f}}^{\prime}$ and write $x=u+v, u \in \mathfrak{A}_{\mathrm{f}} \cap \mathfrak{A}_{\mathrm{g}} v \in \mathfrak{R}_{\mathrm{f}}$ using (2.9). Define $\theta\left(x^{\prime}\right)=u+\mathfrak{F}_{\mathrm{g}}$. This gives an unambiguous definition of a map $\theta: \mathfrak{A}_{\mathfrak{f}}^{\prime} \rightarrow \mathfrak{A}_{\mathrm{g}}^{\prime}$ which is easily shown to be an algebra isomorphism.

If $\mathrm{f} \neq \mathrm{g}$ and $\mathfrak{R}_{\mathrm{f}} \subseteq \mathfrak{R}_{\mathrm{g}}$ then (2.9) implies $\mathfrak{A}_{\mathrm{f}} \subseteq \mathfrak{A}_{\mathrm{g}}$, contradicting Lemma 2.3.

To prove the second last statement in the theorem, first suppose $\mathfrak{R}_{\mathfrak{t}} \cap \mathfrak{R}_{\mathrm{g}}=\{0\}$; then with $x \in \mathfrak{R}_{\mathrm{f}}$ as in (2.11) we get

$$
a \in \mathfrak{A}_{\mathrm{f}} \cap \mathfrak{A}_{\mathrm{g}} \cap \mathfrak{R}=\mathfrak{R}_{\mathrm{f}} \cap \mathfrak{R}_{\mathrm{g}}=\{0\},
$$

whence $x=i b \in i \Re_{g}$. Conversely, if $\Re_{f}=i \Re_{g}$ then $\mathfrak{R}_{\mathrm{f}} \cap \mathfrak{R}_{\mathrm{g}}=\mathfrak{R}_{\mathrm{g}} \cap i \mathfrak{R}_{\mathrm{g}}=\{0\}$.

Finally, if $\mathfrak{A}_{\mathrm{f}}$ is semisimple, that is, $\mathfrak{R}_{\mathbb{f}}=(0)$, then (2.9) gives $\mathfrak{A}_{\mathbb{f}}=\mathfrak{A}_{\mathrm{f}} \cap \mathfrak{A}_{\mathrm{g}} \subseteq \mathfrak{A}_{\mathrm{g}}$, so $f=g$ by Lemma 2.3 . 
REMARK. Equations (2.10) hold also when $f, g$ do not commute. See [6, Lemma 2.1].

\section{EXAMPLES}

3.1. Subalgebras of $C(\Omega, \mathbb{C})$. Let $\Omega$ be a compact Hausdorff topological space, and $\phi$ be any homeomorphism of $\Omega$ which exchanges point, that is $\phi^{2}=i d$. Let $\mathfrak{B}$ be any Banach subalgebra of $C(\Omega, \mathbb{C})$. The norm is the sup norm. Define, for any $x \in$ $C(\Omega, \mathbb{C})$,

$$
(x f)(t):=x(\phi(t))^{-} \quad \text { for all } t \in \Omega .
$$

Then $f$ has the properties (1) to (5) of Section 2. Suppose that $\mathfrak{B}$ is closed under $f$; then $f$ is a conjugation on $\mathfrak{B}$, and its selfconjugate subalgebra $\mathfrak{A}_{\mathfrak{f}}$ is the set of all those functions taking complex conjugate values at paired points, and hence real values at fixed points of $\phi$. If $\phi=$ identity, then $\mathfrak{A}_{\mathrm{f}}$ consists of all the realvalued members in $\mathfrak{B}$. Otherwise, $\mathfrak{A}_{\mathfrak{f}}$ may contain nonreal functions.

3.2. Let $\mathfrak{B}$ be a complex Banach algebra of $n \times n$ matrices. Let $P$ be a matrix satisfying $P P^{-}=I$ (where as usual - denotes complex conjugation), and define $\mathrm{f}$ by

$$
X \mathrm{f}:=P X^{-} P^{-1} \text { for all } X \in \mathfrak{B} \text {. }
$$

Provided $\mathfrak{B}$ is closed under $f, f$ is a conjugation on $\mathfrak{B}$. If $X \mathbf{g}:=Q X^{-} Q^{-1}$, where $Q$ is another such matrix, then $f$ and $g$ are distinct if and only if $Q P^{-}$does not lie in the commutant of $\mathfrak{B}$. On the other hand, $f$ and $g$ commute if and only if $\left(Q P^{-}\right)^{2}$ does lie in the commutant.

3.3. We shall construct an example of a complex Banach algebra $\mathfrak{B}$ carrying two distinct and commuting conjugations $d$ and $f$, each of whose selfconjugate subalgebras is strictly real.

Let $\mathfrak{M}$ be the complex Banach algebra of all $2 \times 2$ matrices over $\mathbb{C}$, with norm say $\|x\|=\max \{|a|+|b|,|c|+|d|\}$ when

$$
x=\left(\begin{array}{ll}
a & b \\
c & d
\end{array}\right) .
$$

Let $\sigma$ be a fixed positive real number, and for the above $x$ write

$$
x \mathrm{c}=\left(\begin{array}{ll}
\bar{a} & \bar{b} \\
\bar{c} & \bar{d}
\end{array}\right), \quad x \mathrm{f}=\left(\begin{array}{cc}
\bar{d} & \sigma^{2} \bar{c} \\
\bar{b} / \sigma^{2} & \bar{a}
\end{array}\right) .
$$

Each of $c$ and $f$ satisfies (1) to (4) and (5') of Section 2, and is therefore a conjugation on $\mathfrak{M}$. Moreover, $c$ and $f$ commute. The $f$-selfconjugate elements are the matrices of the form

$$
z=\left(\begin{array}{cc}
a & \sigma^{2} \bar{c} \\
c & \bar{a}
\end{array}\right)
$$


here $\left.\operatorname{Sp}(z)=\left\{\operatorname{Re}(a) \pm \sqrt{ }(\operatorname{Re}(a))^{2}-|a|^{2}+\sigma^{2}|c|^{2}\right\}\right\}$, so $\operatorname{Sp}(z)$ is real if and only if $|\operatorname{Im}(a)| \leqslant \sigma|c|$. Let $y$ denote an element of the form (3.2), say

$$
y=\left(\begin{array}{cc}
p & \sigma^{2} \bar{q} \\
q & \bar{p}
\end{array}\right)
$$

where $p, q$ are chosen not both real, and to satisfy

$$
|\operatorname{Im}(p)| \leqslant \sigma|q| ;
$$

then

$$
y c \neq y, \quad y \mathrm{f}=y, \quad(y c) \mathrm{f}=y \mathrm{c}, \quad \mathrm{Sp}(y) \cup \mathrm{Sp}(y c) \subset \mathbb{R} .
$$

We make a special choice of $\boldsymbol{p}$ and $q$, namely, let

$$
p=\alpha+i \sigma \delta \text { and } q=i \delta, \text { with } \alpha, \beta, \delta \text { real and nonzero }
$$

(so that equality holds in (3.3)). Then $y=\alpha e+i \delta s, y c=\alpha e-i \delta s$, where $e$ is the $2 \times 2$ unit matrix and

$$
\mathrm{s}=\left(\begin{array}{cc}
\sigma & -\sigma^{2} \\
1 & -\sigma
\end{array}\right)
$$

here $s^{2}=0$; matrices $y$ and $y c$ commute, and together they generate in $\mathfrak{M}$ the real unital Banach subalgebra

$$
\mathfrak{Z}=\{\mu e+i \nu s: \mu, \nu \in \mathbb{R}\}
$$

Since $\operatorname{Sp}(s)=\{0\}, Z$ is strictly real. Moreover, $\mathbb{Z}$ is closed under $c$ and $f$, in fact $f$ relativises to the identity on $\mathfrak{Z}$. Each element of $\mathfrak{Z}$ is of the form (3.2).

Let $\mathfrak{B}$ denote the complex Banach subalgebra of $\mathfrak{M}$ generated by $\mathfrak{Z}$; it consists of all matrices of the form

$$
w=z_{1}+i z_{2},
$$

where $z_{1}, z_{2} \in \mathfrak{Z}$. It is easy to verify that every element $w$ in $\mathfrak{M}$ has a unique representation of the form (3.5) with $z_{1}, z_{2}$ of the form (3.2); therefore for each $w$ in $\mathfrak{B}$ the representation (3.5) with $z_{1}, z_{2} \in \mathfrak{Z}$ is unique. Thus $\mathfrak{B}$ is isomorphic to a Banach complexification of 3 . Moreover, $w \mathrm{f}=z_{1}-i z_{2}$, so $\mathfrak{B}$ is invariant under $\mathrm{f}$, which restricts to a conjugation on $\mathfrak{B}$. We have $w f=w$ if and only if $w \in Z$. Thus the $f$-selfconjugate subalgebra of $\mathfrak{B}$ is

$$
\mathfrak{A}_{\mathrm{f}}=\mathfrak{Z}=\{\mu e+i \nu s: \mu, \nu \in \mathbb{R}\} .
$$

Now there exists also on $\mathfrak{B}$ the conjugation $c$, for which

$$
w \mathrm{c}=z_{1} \mathrm{c}-i z_{2} \mathrm{c}
$$


since by construction $z c \in \mathcal{Z}$ when $z \in \mathcal{Z}$. This $\mathrm{c}$ is distinct from $\mathrm{f}$ on $\mathfrak{Z}$, and therefore on $\mathfrak{B}$. Calculation shows that $w c=w$ if and only if

$$
w=\mu e+\nu s \text { for some real } \mu, \nu ;
$$

for such $w, \operatorname{Sp}(w)=\{\mu\}$. Thus $\mathfrak{A} c=\{\mu e+\nu s: \mu, \nu \in \mathbb{R}\}$ is also strictiy real.

This example is a case of the preceding Example 3.2. It shows incidentally that Theorem 2.10 has nonvoid application. In the notation of that theorem, $\mathfrak{R}_{c}=\cdot \mathbb{R} s$ and $\mathfrak{R}_{\mathrm{f}}=i \mathbb{R} s$.

\section{Plenitude of strictly Real algebras}

This section is devoted to conditions of various kinds ensuring that a Banach algebra is strictly real, and to gathering evidence to show that strictly real Banach algebras are of reasonably frequent occurrence in nature. For interest, we recall some results of Ingelstam.

THEOREM 4.1. (Ingelstam [2]) Each of the following conditions is separately sufficient for a real Banach algebra $\mathfrak{A}$ to be strictly real:

(a) For every $x \in \mathfrak{A}, e+x^{2}$ is invertible in $\mathfrak{A}$.

(b) For every $x \in \mathfrak{A}$, the function $\lambda \mapsto \exp \left(-\lambda x^{2}\right), \mathbb{R}^{+} \rightarrow \mathfrak{A}$ is bounded.

(c) For every $x \in \mathfrak{A}$, each ray from $e$ of the form $\left\{e-\lambda x^{2}: \lambda \geqslant 0\right\}$ is in the enveloping cone at $e$.

(d) For every $x \in \mathfrak{A}, \lim _{\lambda \rightarrow 0} \lambda^{-1}\left(\left\|e-\lambda x^{2}\right\|-1\right) \leqslant 0$.

Of these conditions, (a) is also necessary.

Another condition, similar to and deducible from (a), but applicable to an algebra which may a priori be complex, is:

Proposition 4.2. ([5]) $A$ Banach algebra $\mathfrak{A}$ is strictly real if and only if, for every $x \in \mathfrak{A}, x^{2} \succeq 0$.

The superficial similarity of conjugations and involutions suggests the next result.

THEOREM 4.3. Let $\mathfrak{B}$ be a complex Banach algebra, and $f$ a conjugation on $\mathfrak{B}$ such that $\|x \mathrm{f} x\|=\|x\|^{2}$ for all $x \in \mathfrak{B}$. Then $\mathfrak{A}_{\mathrm{f}}$, the selfconjugate subalgebra of $\mathrm{f}$, is strictly real.

The proof is an almost verbatim repetition of an analogous argument for $C^{*}$ algebras: see [3, p.238, Proposition 4.1.1]. It starts with the useful fact that $\nu(x)=\|x\|$ for every $x$ for which $x f x=x(x)$.

THEOREM 4.4. The class of strictly real Banach algebras is a variety, in the sense:

(i) Every Banach subalgebra of a strictly real Banach algebra is strictly real. 
(ii) Let $\mathfrak{A}$ be a strictly real Banach algebra and let $H$ be an algebra homomorphism from $\mathfrak{A}$ into a real Banach algebra $\mathfrak{D}$, with closed range $H(\mathfrak{A})$. Then $B(\mathfrak{A})$ is strictly real.

(iii) Let $\mathfrak{D}$ and $\mathfrak{E}$ be strictly real Banach algebras; then their direct sum $\mathfrak{D} \oplus \mathfrak{E}$ is strictly real.

Proof: (i) If $\mathfrak{A}$ is strictly real and $\mathfrak{D}$ is a Banach subalgebra of it, we have $\mathrm{Sp}_{\mathfrak{D}}(x)=\mathrm{Sp}_{\mathfrak{A}}(x) \subset \mathbb{R}$ for every $x \in \mathfrak{D}$, by $[1, \mathrm{p} .25$, Proposition 12].

(ii) $H(\mathfrak{A})$ is a Banach subalgebra of $\mathfrak{D}$, having an identity $H(e)$. We extend $H$ to a homomorphism $H^{*}: \mathfrak{A}_{\mathbb{C}} \rightarrow \mathfrak{D}_{\mathbb{C}}$ between the complexifications by defining $H^{*}(a+i b)=$ $H(a)+i H(b)$. Then $H^{*}\left(\mathfrak{A}_{\mathbb{C}}\right)=(H(\mathfrak{A}))_{\mathbb{C}}$ is a Banach subalgebra of $\mathfrak{D}_{\mathbf{C}}$. For any $x \in \mathfrak{A}$ we have

$$
\operatorname{Sp}_{H(\mathfrak{x})_{\mathbb{C}}}(H(x)) \subseteq \mathrm{Sp}_{\mathfrak{x}_{\mathrm{C}}}(x),
$$

so $H(x)$ has real spectrum.

(iii) $\mathfrak{D} \oplus \mathfrak{E}$ is defined to be the vector-space direct sum, made into a real normed algebra by writing

$$
\begin{aligned}
& \left(x_{1}, y_{1}\right) \cdot\left(x_{2}, y_{2}\right):=\left(x_{1} x_{2}, y_{1} y_{2}\right), \\
& \|(x, y)\|:=\max \left\{\|x\|_{\mathcal{D}},\|y\|_{\mathcal{E}}\right\} .
\end{aligned}
$$

These ensure that $\mathfrak{D} \oplus \mathfrak{E}$ is a real Banach algebra. It is routine to verify that

$$
\mathrm{Sp}_{\mathfrak{D} \oplus \mathbb{E}}((x, y))=\mathrm{Sp}_{\mathfrak{D}}(x) \cup \mathrm{Sp}_{\mathfrak{E}}(x),
$$

which implies the result.

We remark that (i) ensures that the definitions (1.1) of the orders are independent of the containing algebra. In (ii) we find that $H$ preserves both orders, that is, every homomorphism is positive. It is not required that $H$ be bounded, nor that $H$ map the identity to the identity. In extension of (iii), one easily shows that a direct sum of arbitrarily many strictly real summands is also strictly real.

We turn to ways in which strictly real algebras may be generated. An element $y$ in a Banach algebra $\mathfrak{B}$ (real or complex) will be called strictly real if $\mathrm{Sp}(y) \subset \mathbb{R}$. A subset $Z$ of $\mathfrak{B}$ will be called quasicommuting if $x y-y x$ belongs to the radical of $\mathfrak{B}$ for every pair of elements $x, y$ in $Z$.

Lemma 4.5. Let $\mathfrak{B}$ be a real or complex Banach algebra, and let $y \in \mathfrak{B}$. Let $\mathfrak{Y}$ be the real Banach subalgebra generated by $y$ and $e$. Then $y$ is strictly real in $\mathfrak{B}$ if and only if $\mathfrak{Y}$ is a strictly real Banach algebra.

Proof: Suppose $\mathfrak{B}$ is real. Then $\mathfrak{Y}_{\mathfrak{C}}$ is a complex Banach subalgebra of $\mathfrak{B}_{\mathbb{C}}$ by virtue of $(2.1)$. If $\mathfrak{Y}$ is strictly real, then $\operatorname{Sp}_{\mathfrak{B}_{\mathfrak{C}}}(y) \subseteq \operatorname{Sp}_{\mathfrak{Y}}(y) \subset \mathbb{R}$, so $y$ is strictly 
real. Conversely, assume $y$ is strictly real. The elements of $\mathfrak{Y}, \mathfrak{Y}_{\mathbf{c}}$ consist of all limits of sequences of polynomials in $y$ with respectively real, complex coefficients. By assumption $\operatorname{Sp}_{\mathfrak{B}_{\mathbf{C}}}(y) \subset \mathbb{R}$, so $\operatorname{Res}_{\mathfrak{B}_{\mathbf{C}}}(y)$ is connected; therefore ([1, p.25, Proposition 14]) $\operatorname{Sp}_{\mathfrak{Y}_{\mathbf{C}}}(y)=\mathrm{S}_{\mathfrak{B}_{\mathbf{C}}}(y)$, whence $\mathrm{Sp}_{\mathfrak{Y}_{\mathbf{C}}}(p)$ is real for every real polynomial $p$ in $y$.

Let $f \in \mathfrak{Y}, f=\lim p_{n}$ for some sequence $\left(p_{n}\right)$ of real polynomials in $y$. Since $\mathfrak{Y}_{\mathbb{C}}$ is commutative, $\mathrm{S}_{\mathrm{P}_{\mathfrak{C}}}$ is lower semicontinuous. Suppose that $\mathrm{Sp}_{\mathfrak{Y}_{\mathbf{C}}}(f)$ contains a number $\alpha+i \beta, \beta \neq 0$. If $U$ denotes the open disc in $\mathbb{C}$ with centre 0 and radius $|\beta|$, there exists an $n$ with

$$
\mathrm{Sp}_{\mathfrak{Y}_{\mathbb{C}}}(f) \subset \operatorname{Sp}_{\mathfrak{Y}_{\mathbb{C}}}\left(p_{n}\right)+U
$$

and this produces a contradiction. Thus $\operatorname{Sp}_{\mathfrak{y}_{\mathbf{C}}}(f)$ is real, that is, $\mathfrak{Y}$ is strictly real.

If instead $\mathfrak{B}$ is a complex algebra, then replace $\mathfrak{B}_{\mathbb{C}}$ by $\mathfrak{B}$ in the above proof.

Lemma 4.6. Let $\mathfrak{B}$ be a real or complex Banach algebra, and let $\left(x_{n}\right)_{n \in \mathbb{N}}$ be a convergent sequence of pairwise quasicommuting, strictly real elements of $\mathfrak{B}$, with limit $\boldsymbol{x}$. Then $\boldsymbol{x}$ is strictly real in $\mathfrak{B}$.

Proof: Suppose first that $\mathfrak{B}$ is a real algebra, and that the terms of the sequence are pairwise commuting. Let $\mathfrak{X}$ be the real Banach subalgebra generated by the terms of the sequence (with $e$ ), so that $x \in \mathfrak{X}$, and $\mathfrak{X}$ is commutative. Assume that there exists a number $\alpha+i \beta, \beta \neq 0$, in $\operatorname{Sp}_{x}(x)$. Arguing from lower semicontinuity as in the proof of Lemma 4.5, we can find an $n$ such that $\operatorname{Sp}_{\mathfrak{X}}(x) \subset \mathrm{Sp}_{\mathfrak{X}}\left(x_{n}\right)+U$, and therefore $|\alpha+i \beta-\gamma|<|\beta|$ for some $\gamma \in \mathrm{Sp}_{x}\left(x_{n}\right)$. For this $n$ let $\mathfrak{E}$ be the real Banach subalgebra of $\mathfrak{X}$ generated by $x_{n}$ and $e$. Lemma 4.5 shows that $\mathfrak{E}$ is strictly real; thus

$$
\mathrm{Sp}_{\mathfrak{X}}\left(x_{n}\right) \subseteq \mathrm{Sp}_{\mathfrak{E}}\left(x_{n}\right) \subset \mathbb{R},
$$

so $\gamma \in \mathbb{R}$, which contradicts the inequality. Therefore $\operatorname{Sp}_{\mathfrak{X}}(x)$ is real. But $\mathbf{S p}_{\mathfrak{B}}(x) \subseteq$ $\mathrm{Sp}_{\mathfrak{X}}(x)$, so $\mathrm{Sp}_{\mathfrak{B}}(x)$ is real.

The case where $\mathfrak{B}$ is complex is proved in the same way.

If more generally the terms of the sequence are only quasicommuting, then we apply the above result in the algebra $\mathfrak{B}^{\prime}:=\mathfrak{B} \bmod \mathfrak{A}, \mathfrak{P}$ being the radical of $\mathfrak{B}$ : in this algebra the terms of the sequence $\left(x_{n}^{\prime}\right)$ commute (Corollary 2.7) and the sequence converges to $x^{\prime}$, so we get $\mathrm{Sp}_{\mathfrak{B}^{\prime}}\left(x^{\prime}\right) \subset \mathbb{R}$. But $\mathrm{Sp}_{\mathfrak{B}}(x)=\mathrm{S}_{\mathfrak{P}^{\prime}}\left(x^{\prime}\right)$.

From the lemmas we obtain

THEOREM 4.7. Let $\mathfrak{B}$ be a real or complex Banach algebra. If $Z$ is any quasicommutative subset of strictly real elements of $\mathfrak{B}$, then $\mathbf{Z}$, the real Banach subalgebra of $\mathfrak{B}$ generated by $Z$, is strictly real.

Proof: Let alg $(Z)$ denote the real subalgebra of $\mathfrak{B}$ generated by $Z$ and $e$; its closure is $\mathcal{Z}$. If $x, y \in Z$ then since $x^{\prime}, y^{\prime}$ commute in $\mathfrak{B}^{\prime}$ we have

$$
\mathrm{Sp}_{\mathfrak{B}}(x+y)=\mathrm{Sp}_{\mathfrak{B}^{\prime}}\left(x^{\prime}+y^{\prime}\right) \subseteq \mathrm{S}_{\mathfrak{B}^{\prime}}\left(x^{\prime}\right)+\mathrm{Sp}_{\mathfrak{B}^{\prime}}\left(y^{\prime}\right)=\mathrm{S}_{\mathfrak{B}}(x)+\mathrm{S}_{\mathfrak{B}}(y) \subset \mathbb{R},
$$


so $x+y$ has real spectrum; so has $x y$, by the same argument. Therefore every element of $\operatorname{alg}(Z)$ is strictly real. Moreover $\operatorname{alg}(Z)$ is quasicommutative, since $Z$ is. Now let $w \in \mathcal{Z}$, with $w$ the limit of a sequence of elements from alg $(Z)$. Lemma 4.6 shows that $S_{P_{\mathfrak{B}}}(w) \subset \mathbb{R}$. Let $\mathfrak{W}$ be the real Banach subalgebra generated by $w$ and $e$. By Lemma 4.5, $\mathrm{Sp}_{\mathfrak{w}}(w)$ is real; also $\mathrm{Sp}_{3}(w) \subseteq \mathrm{Sp}_{\mathfrak{w}}(w)$, so $\mathrm{Sp}_{3}(w)$ is real. This proves that $\mathfrak{Z}$ is strictly real.

COROLlary 4.8. Let $\mathfrak{B}$ be an Hermitian Banach star algebra. If $F$ is any quasicommutative subset of the set of selfadjoint elements of $\mathfrak{B}$, then the real Banach star subalgebra generated by $F$ and $e$ is strictly real.

Proof: By definition $[1, p .224] \mathfrak{B}$ is a complex Banach algebra with involution, written ${ }^{*}$ say, in which $\operatorname{Sp}(x) \subset \mathbb{R}$ whenever $x^{*}=x$. First suppose that $F$ is commutative. Let $\mathfrak{F}$ be the real Banach subalgebra of $\mathfrak{B}$ generated by $F$ and $e$; by Theorem $4.7, \mathfrak{F}$ is strictly real. But $\mathfrak{F}$ consists of limits of real polynomials in elements of $F$; these limits are selfadjoint elements and hence $\mathfrak{F}$ is in fact the real Banach *-subalgebra generated by $F$ and $e$.

Suppose instead that $F$ is quasicommutative. Form $\mathfrak{B}^{\prime}=\mathfrak{A} \bmod \mathfrak{R}$, which has $\mathfrak{F}^{\prime}:=\left\{f^{\prime}: f \in F\right\}$ as a commutative subset. $\mathfrak{B}^{\prime}$ is made into a star subalgebra by the involution

$$
\left(x^{\prime}\right)^{\star}:=x^{*^{\prime}}=x^{*}+\mathfrak{R} \text {. }
$$

(To prove that $\star$ is well-defined we use the formula $R=\left\{x: \nu\left(x^{*} x\right)=0\right\}$ (see $[1$, Theorem 9, p.227]) to show that $\mathfrak{R}$ is closed under ${ }^{*}$.) In fact $\star$ makes $\mathfrak{B}^{\prime}$ into an Hermitian star algebra. For let $x^{\prime} \in \mathfrak{B}^{\prime}$ and $x^{\star} \star=x^{\prime}$. Write $x=p+i q, p=$ $\left(x+x^{*}\right) / 2, q=i\left(x^{*}-x\right) / 2$. Then $p^{*}=p$ and $q \in \mathfrak{R}$ since $\left(x^{*}-x\right)^{\prime}=0^{\prime}$, whence $\mathrm{Sp}_{\mathfrak{B}^{\prime}}\left(x^{\prime}\right)=\mathrm{Sp}_{\mathfrak{B}}(x)=\mathrm{Sp}_{\mathfrak{B}}(p) \subset \mathbb{R}$. Also every element of $F^{\prime}$ is self-adjoint with respect to $\star$. Thus by applying the commutative case to $\mathfrak{B}^{\prime}$ and $F^{\prime}$ we conclude

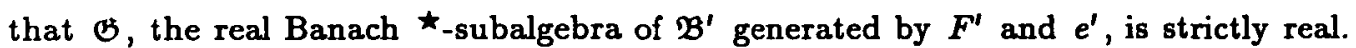
Consider the set

$$
\mathfrak{H}:=\left\{x \in \mathfrak{B}: x^{\prime} \in \mathfrak{G}\right\} .
$$

This is a real Banach subalgebra of $\mathfrak{B}$, moreover $x \in \mathfrak{H}$ if and only if $x^{*} \in \mathfrak{H}$, and $F \cup\{e\} \subseteq \mathfrak{H}$. Thus the real Banach star subalgebra, $\mathfrak{E}$ say, of $\mathfrak{B}$ generated by $F, e$ is a Banach subalgebra of $\mathfrak{H}$. Now

$$
\operatorname{Sp}_{\mathfrak{f}}(x)=\operatorname{Sp}_{\mathfrak{H}^{\prime}}\left(x^{\prime}\right) \subset \mathbb{R} .
$$

Thus $\mathfrak{H}$ is strictly real, and therefore $\mathfrak{E}$ is strictly real, by Theorem 4.4 (i).

$C^{*}$-algebras are Hermitian (see [3, p.238]), therefore Corollary 4.8 applies in particular to them. The $B^{*}$ property is $\left\|x^{*} x\right\|=\|x\|^{2}$, so here we have a result superficially like Theorem 4.3, but no conjugation operator is invoked in Theorem 4.7 or its corollary. 


\section{Conclusion}

Strictly real Banach algebras form a well delineated and manageable class of quasicommutative Banach algebras.

\section{REFERENCES}

[1] F.F. Bonsall and J. Duncan, Complete normed algebras (Springer-Verlag, Berlin, Heidelberg, New York, 1973).

[2] L. Ingelstam, 'Real Banach algebras', Ark. Mat. 5 (1964), 239-270.

[3] R.V. Kadison and J.R. Ringrose, Fundamentals of the theory of operator algebras, Vol 1. (Academic Press, New York, 1983).

[4] I. Kaplansky, 'Normed algebras', Duke Math. J. 16 (1949), 399-418.

[5] J.B. Miller, 'The natural ordering on a strictly real Banach algebra', Math. Proc. Cambridge Philos. Soc. 107 (1990), 539-556.

[6] J.B. Miller, 'Analysis in strictly real Banach algebras', Monash Univ. Analysis Paper No.70 (Monash University, Clayton, Australia).

[7] C. Rickart, General theory of Banach algebras (Van Nostrand, Princeton, 1960).

Department of Mathematics

Monash University

Clayton, Victoria 3168

Australia 